\title{
LA ESTRATEGIA DE INTEGRACIÓN DE ESPACIOS ABIERTOS Y PATRIMONIO EN EL PLAN METROPOLITANO DE DESARROLLO URBANO LIMA-CALLAO 2035
}

\author{
OPEN SPACE AND HERITAGE INTEGRATION STRATEGY IN THE 2035 METROPOLITAN LIMA- \\ CALLAO URBAN DEVELOPMENT PLAN
}

\author{
JUAN MANUEL DEL CASTILLO CÁCERES ${ }^{(\star \star)} Y$ MARIANELA CASTRO DE LA BORDDA ${ }^{[\star \star \star]}$ \\ Fecha de recepción: 07 de setiembre de 2015 \\ Fecha de aprobación: 29 de setiembre de 2015
}

\section{RESUMEN}

La innovadora estrategia para integrar los espacios abiertos y el patrimonio arqueológico de la ciudad de Lima como parte de una red de corredores verdes para el disfrute de la comunidad es uno de los mayores aportes del Plan Metropolitano de Desarrollo Urbano Lima-Callao 2035 (PLAM2035). La mayor parte de este patrimonio arqueológico se encuentra en la periferia de la ciudad, vinculado a los valles de los ríos y lomas, convertido en basurales o espacios residuales amenazados por la urbanización informal. Sin embargo, es allí mismo donde reside su gran potencial de convertirse en grandes espacios ecológico-culturales que conecten los ecosistemas de nuestra metrópolis al patrimonio histórico, para de esta forma brindarle protección, y fomentar el turismo, la investigación local y foránea, y el encuentro de la ciudadanía. Este estudio busca analizar las estrategias de integración entre el Sistema de Espacios Abiertos e Infraestructura Ecológica, y la Red de Patrimonio y Paisaje, enfocándose en su contexto, su nueva visión conceptual y su proceso.

\section{PALABRAS CLAVE}

Espacios abiertos, patrimonio andino, planeamiento urbano

\begin{abstract}
One major contribution of the Metropolitan Lima Urban Development Plan 2035 (PLAM2035) to the history of planning is its innovative strategy to integrate the city's open spaces and archaeological heritage within a network of green corridors for the enjoyment of the community. Most of Lima's architectural heritage is located in the periphery of the metropolis, linked to river valleys and mountains, sites that have been converted into landfills or wastelands threatened by informal urbanisation. However, these places foster the potential to become ecological and cultural assets that help in connecting the metropolitan ecosystems with Andean heritage, to promote the protection of archaeological sites, tourism, national and international research activities, and community integration. This study seeks to analyse the PLAM2035's proposal regarding the integration between the System of Open Spaces and Ecological Infrastructure and the Heritage and Landscape Network focusing on its context, its new conceptual vision, and its process.
\end{abstract}

\section{KEYWORDS}

Open spaces, Andean heritage, urban planning

(*) Este artículo es parte de la experiencia de ambos autores como miembros del equipo técnico del PLAM2035; las exploraciones académicas realizadas en la UPC en el Taller de Diseño Arquitectura e Identidad; una propuesta de doctorado aprobada por la Universidad de Manchester; un artículo y conferencia presentados en Montevideo-Uruguay; y un proyecto de investigación actual en la UPeU en Ñaña.

(**) Máster con honores en Arquitectura y Urbanismo, Universidad de Manchester, Reino Unido. Arquitecto, PUCP. Planificador urbano, investigador y docente de la Universidad Peruana Unión, Universidad Peruana de Ciencias Aplicadas y Universidad Científica del Sur. En la actualidad, dirige proyectos de investigación en los paisajes culturales de Ñaña y Mangomarca-Campoy, en la margen derecha del Valle del Rímac. Fue miembro del equipo territorial del PLAM 2035, donde participó en la elaboración del Diagnóstico Medio Ambiental y el Sistema de Espacios Abiertos e Infraestructura Ecológica. Coautor del libro Memoria Territorial y Patrimonial: Artes y Fronteras. Contacto: juandelcastillo@upeu.edu.pe

$\left.{ }^{* * *}\right)$ Máster en Vivienda y Urbanismo, Architectural Association, Londres. Arquitecta UPC. Cofundadora de FAN-CY STUDIO LIMA, oficina de investigación, diseño y construcción. Es Docente universitaria de Talleres de Diseño y de Cursos de Investigación en la Universidad de Lima, en la Universidad Peruana de Ciencias Aplicadas y en la Universidad Científica del Sur. A partir de una búsqueda multiescalar, le interesa experimentar los límites entre la ciudad y la arquitectura a través de la investigación y del desarrollo de estrategias proyectuales en equipos multidisciplinares. Fue parte del equipo técnico del Plan de Espacios Abiertos e Infraestructura Ecológica, parte del PLAM 2035. Contacto: marianela@fancystudiolima.com 
El objetivo de este estudio es analizar las estrategias de integración entre el Sistema de Espacios Abiertos e Infraestructura Ecológica, y la Red de Patrimonio y Paisaje en el PLAM2035. La investigación explora el potencial de establecer una estrategia que relacione los actuales espacios abiertos públicos de la ciudad (parques, plazas y calles), los que resulten de la incorporación de parte de la estructura ecológica de Lima (ríos, valles, lomas, humedales, litoral) y su patrimonio histórico, con el objetivo de lograr su posterior protección y preservación; el aumento de la dotación de áreas verdes de amortiguamiento y uso público; y su articulación con un sistema de corredores verdes que lo relacione con los ecosistemas urbanos existentes, para proteger y aumentar la biodiversidad urbana.

Este escrito revisa los principales conceptos relacionados a la puesta en valor del patrimonio cultural, los espacios abiertos de uso público y la regeneración de los ecosistemas urbanos. Por otro lado, el ensayo también brinda una aproximación a la historia del territorio de Lima que permita entender mejor su naturaleza y derribar viejos estigmas académicos sobre su actual conformación. Así, se concibe la relación ancestral entre el patrimonio arqueológico y los ecosistemas de Lima como una vieja continuidad a ser restaurada, con miras a incorporar a la estructura urbana nuevos espacios abiertos que sirvan como escenario para la integración social y la cultura viva.

Además, el contexto en el que se generó el PLAM2035 es motivo de estudio para brindar una visión general del marco legal y técnico en el cual se inscriben las iniciativas descritas, las cuales forman parte de procesos de ordenamiento territorial y urbanístico que deben ser promovidos para generar mayor institucionalidad en nuestro medio.

La visión conceptual contemporánea con la cual se abordan los objetivos y lineamientos para los espacios abiertos en el PLAM2035 será también explorada, haciendo hincapié en el patrimonio arqueológico como huella del equilibrio ambiental logrado por el manejo territorial ancestral.

\section{Teorizando el Espacio Abierto y el Patrimonio}

La reflexión sobre el concepto de espacio abierto y su relación con la vida pública adquiere cada vez mayor importancia en el siglo XXI. Por un lado, debido al creciente interés académico que se manifiesta en publicaciones y la fundación de centros de investigación sobre el tema. Por otro lado, debido a su relación con conceptos como naturaleza, biodiversidad, activismo ecológico y político, calidad de vida y ciudadanía, se incluye con cada vez mayor frecuencia en estudios y estrategias de espacios abiertos para áreas metropolitanas a nivel global.

Una actitud hacia la naturaleza y el deseo de entrar en contacto con ella están contenidos en los valores sociales y culturales del espacio abierto, mientras que el entendimiento contemporáneo de la ecología nos lleva a intuir nuevas formas de servir tanto a las necesidades humanas como al marco ecológico más amplio de la estructura de espacios abiertos urbanos (Ward Thompson, 2002). Al mismo tiempo, la idea de los paisajes de naturaleza salvaje que nos rodean han pasado de ser considerados opuestos a la civilización occidental a un estado de tesoro, plasmado en la fundación del movimiento de Parques Nacionales (Jorgensen \& Tylecote, 2007). El uso figurado de 'naturaleza salvaje'y sus palabras de compañía enfatiza su papel como territorio psicológico y social (o más bien asocial), así como geográfico.

Sin embargo, la investigación sobre espacios intersticiales entre el 'completo salvajismo' y las ciudades sigue siendo problemática, en parte porque las estrategias de gestión de la naturaleza urbana no tienen en cuenta las conexiones entre sistemas ecológicos y áreas urbanas (Pickett et al., 1997) y, por tanto, no integran a los seres humanos como componentes de los ecosistemas (Alberti et al., 2003). 
En el caso de áreas metropolitanas latinoamericanas como Lima, si bien es cierto que la urbanización informal generalmente ha sido identificada como una amenaza para la biodiversidad (Benítez, Pérez-Vásquez, Nava-Tablada, Equihua \& Álvarez-Palacios, 2012) y los monumentos históricos, es crucial entender las comunidades periurbanas donde existe evidencia de los vínculos entre su patrimonio ancestral y la regeneración potencial de sus ecosistemas. En ese sentido, es importante superar las divisiones académicas occidentales rígidas entre naturaleza y ciudad, para observar más profundamente el contexto socioecológico de sus espacios intersticiales y, asimismo, explorar el papel de los restos arquitectónicos ancestrales como elemento de antigua integración entre ambos dominios (Del Castillo, 2015).

Por otro lado, las actitudes hacia la naturaleza en el Sur Global, específicamente en la región andina de América Latina, siempre han conservado sus lazos ancestrales entre lo humano, el entorno natural y lo sobrenatural. El reconocimiento de la Madre Tierra (Pachamama, en quechua) como un sistema dinámico vivo, lo cual le otorga derechos legales integrales comparables a los derechos humanos en Bolivia, es solo un ejemplo de esta filosofía (Buck, 2012). Sin embargo, la falta de implementación de políticas públicas en espacios abiertos, que se traduce en una gran deficiencia de infraestructura ecológica adecuada, amenaza los grandes ecosistemas en las metrópolis contemporáneas de América Latina, donde las fronteras no son fijas, sino que están continuamente reconfigurándose. Hoy en día la distinción occidental tradicional entre el medio ambiente y la sociedad, entre la naturaleza y la cultura, se vuelve cada vez más conflictiva, ambigua y problemática (Swyngedouw, 2010).

Así mismo, el patrimonio arqueológico y arquitectónico guarda especial relación con los espacios abiertos públicos como parques urbanos, plazas y calles, los cuales en ciertos casos proveen resguardo y amortiguamiento para los monumentos históricos frente a las distintas actividades humanas que puedan resultarles dañinas. El patrimonio es también un símbolo de identidad e integración con la naturaleza y, por tanto, en realidades tan difíciles como la peruana, es testigo del activismo ciudadano por resguardar nuestra historia, y reencontrarnos con nuestra herencia cultural andina y su sabiduría ancestral, ante el abandono de las autoridades competentes.

Entendemos como patrimonio a los bienes que heredamos de nuestros ascendientes o que traspasamos en herencia. Estos se encuentran acotados en espacio y tiempo, son mutables y su significación (al implicar valores y cosmovisiones distintas) es un concepto relativo en sí mismo. Por cultura nos referimos a la producción de fenómenos que ayudan a entender, reproducir o transformar un sistema social, siendo esta aprendida y parte de la socialización humana. El resultado de la unión de estos dos conceptos es el de patrimonio cultural, por el cual se entiende un conjunto de elementos susceptibles de ser estimados por un ente social como testimonios de la actividad humana, y comprende al conjunto de actuaciones programadas para optimizar su conservación y uso adecuado a las exigencias sociales contemporáneas (Hayakawa, 2010). Esto implica una serie de apuestas estratégicas: turismo cultural, concertación, subsidiariedad, planificación, transversalidad y desarrollo, sistemas de presentación y proyectos de desarrollo local. En este sentido es clave el tener una estrategia como inicio, de naturaleza mutable y que sea coherente con acciones concretas, lograr sostenibilidad en el tiempo para los proyectos y que exista una apropiación social del patrimonio cultural (Hayakawa, 2012).

En el caso de Lima, Perú, la falta de planificación ante los procesos de migración durante los últimos 30 años no solo ha puesto en peligro los ecosistemas que proveen el soporte ecológico de la ciudad, sino que también el patrimonio arqueológico se encuentra bajo constante amenaza de destrucción. La falta de un programa nacional de vivienda barata que atienda el inmenso déficit en los sectores populares, y la carencia de políticas de protección y puesta en valor de estos espacios, los hace presa fácil de mafias de terrenos que abundan en los márgenes de la ciudad, y se aprovechan de la demanda para ocupar ilegalmente, lotizar y vender espacios inhabitables (Riofrío \& Cabrera, 2010). 


\section{El hombre y el territorio de Lima}

Las transformaciones del modelo territorial de Lima, desde su fundación española hasta nuestros días, pueden resumirse en una curva creciente hacia un uso del suelo cada vez más deficiente, como resultado de una estructura urbana concebida como artificio, cuyo emplazamiento significó una imposición importada en la geografía natural y una relación de explotación insostenible de los ecosistemas existentes, más que de integración a ellos. Esta forma occidental de hacer ciudad, que buscaba separar claramente los dominios del hombre como algo externo a la naturaleza y, por lo tanto, establecer la contraposición urbano-rural/costa-sierra, 'desertificó poco a poco el área de valle que ocupó, mientras que su posterior desarrollo urbano (catalizado por la falta de planificación y contaminación) ha logrado el efecto psicológico de invisibilizar su estructura ecológica para una porción considerable de sus habitantes.

Una aproximación hacia el territorio de Lima con mayor grado de interdisciplinariedad quizás pueda ayudarnos a disolver esta nebulosa posada sobre su naturaleza.

\section{Lima la Andina}

La implantación del damero de Lima establece un antes y un después en la utilización del territorio peruano.

Antes de su fundación, el complejo sistema de poblados indígenas asentados a lo largo del valle combinaba la economía agrícola con la caza y recolección en las lomas cercanas (llenas de vegetación y fauna), y con el intercambio de productos con pastores de las tierras altas, hacia el este, y con pescadores, hacia el oeste.

Como en la mayoría de valles de la costa, la actividad agrícola concentraba a los habitantes en las proximidades del valle en asentamientos de escala controlada (para maximizar la superficie de cultivo). En asentamientos mayores apareció la arquitectura monumental entre 1400 y 600 a.C. , que se manifestaba en 'parejas' y grupos 'jerarquizados' a ambas márgenes del río. En el caso de los ríos Chillón y Rímac, sus valles parecen haber formado parte de un mismo sistema (Kaulicke, 2008).

Las evidencias de un territorio rico en recursos naturales y, por lo tanto, antítesis del desierto, no tardan en aparecer en monumentos como Garagay, por ejemplo, donde aparecen diferentes especies marinas en gran cantidad; evidencias botánicas de maní, frijoles, haba, calabaza, zapallo, la presencia masiva de la lúcuma -fruto de un árbol que crece aún hoy en día en las lomas-, y otros frutos como pacae, palta y guayaba. Así mismo, se han hallado restos de plantas como totora, grama, carrizo, caña brava y junco, que indican un ambiente húmedo, distinto al actual; no se excluye que se hayan utilizado pantanos, puquios y lomas para una agricultura que no dependía de un sistema de complejos canales de irrigación en el formativo medio (Kaulicke, 2008). Posteriormente, con mayor tecnología, estos asentamientos basarían los límites territoriales en la administración del sistema de riego existente.

Este sistema de asentamientos fuertemente conectados se inscribía, a escala mayor, en una estrategia basada en el establecimiento de sinergias entre asentamientos de diferentes pisos altitudinales (Lumbreras, 2008). Notablemente administrada durante el Tawantinsuyu, esta permitía superar las dificultades impuestas por el agreste territorio peruano. Tal organización espacial, sin embargo, no puede ser considerada como un antecedente directo de la Lima actual, puesto que la Colonia reemplazó el modelo cooperativo-asociativo existente (policéntrico) por un modelo de concentración del poder económico, político y cultural (monocéntrico).

La fundación española explotó colonialmente esta preexistencia con innumerables recursos naturales, de los cuales dependía una red económica. Al implantar un modelo centralista, debido al crecimiento de la capital, Lima, que consumía recursos desme- 
TAWANTINSUYU

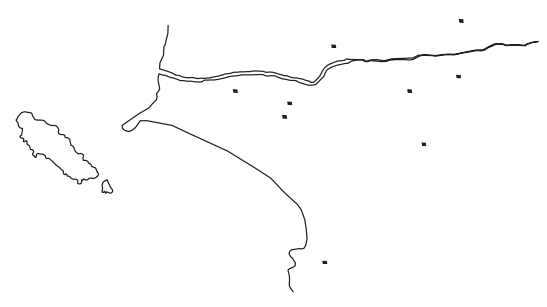

\section{COLONIA}

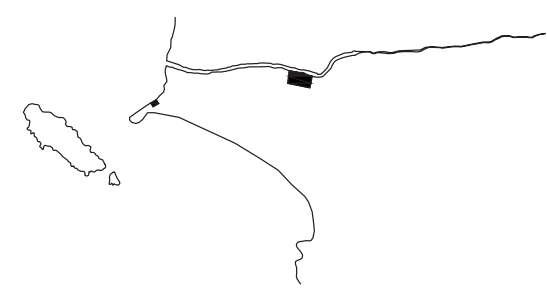

0 $10 \mathrm{~km}$

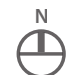

didamente en desmedro del resto de asentamientos, sujeta a mayor escala a una metrópolis externa (Madrid), muchas de estas actividades y sus núcleos desaparecieron, o debieron someterse a la nueva lógica espacial para sobrevivir.

Sin embargo, Lima jamás fue concebida como una ciudad del desierto. Si nos remontamos al momento de su fundación en 1535, esta se estableció sobre un valle copioso. Un paisaje cultural admirable y con innumerables recursos fue la razón principal por la que Pizarro escogió esta ubicación para la capital. Dichas razones aparecen en las descripciones del primer invasor cronista, Pedro Cieza de León: "El valle de Lima es el mayor y más ancho de todos los que se han escripto de Tumbez a él; y así, como era grande, fue muy poblado" (1550, p. 283). A escala urbana, su interior también distaba muchísimo de un ambiente desértico:

Esta ciudad, después del Cuzco, es la mayor de todo el reino del Perú y la más principal, y en ella hay muy buenas casas, y algunas muy galanas con sus torres y terrados, y la plaza es grande y las calles anchas, y por todas las más de las casas pasan acequias, que es no poco contento; del agua dellas se sirven y riegan sus huertos y jardines, que son muchos, frescos y deleitosos. (Cieza de León, 1550, p. 284)

Ni siquiera es posible afirmar que los alrededores inmediatos de Lima eran desérticos:

Fuera de la ciudad, a una parte y a otra, hay muchas estancias y heredamientos, donde los españoles tienen sus ganados y palomares, y muchas viñas y huertas muy frescas y deleitosas, llenas de las frutas naturales de la tierra y de higueras, platanales, granados, cañas dulces, melones, naranjos, limas, cidras, toronjas, y las legumbres que se han traído de España; todo tan bueno y gustoso que no tiene falta, antes digo por su belleza para dar gracias al gran Dios y señor Nuestro, que lo crió. (Cieza de León, 1550, p. 284)

Asistimos entonces al despliegue de un rico paisaje cultural, sobre el cual las Leyes de Indias decretaban implantar una propuesta urbanística radicalmente racionalista: la geometría pura de un rectángulo sobre una gigantesca alfombra de vegetación (Del Castillo, 2015).

\section{Lima la Desbordante}

El proceso de invasión española, y sus continuas y largas guerras significaron un holocausto, no solo para la población del Tawantinsuyu, que se había reducido de aproximadamente 9 millones a solo 600 mil habitantes hacia 1620 (Cook, 1981), sino también para los bosques de Lima, que desaparecieron en 1535 (Buenaño, 2000). Sin embargo, pasado este período, en la Colonia la estructura demográfica y urbana tuvo cierta estabilidad. Esta no cambió significativamente hasta la República, hacia la década de 1850, con la aparición de los ferrocarriles a vapor y, luego, hacia 1870,
Figura 1. Modelo lineal andino vs. modelo nuclear europeo

Fuente. Elaboración propia a partir de Canziani, J. (2009). Ciudad y territorio en los Andes. Lima, Perú: Fondo Editorial PUCP y Gunther, J. (1983). Planos de Lima 1613-1983. Lima, Perú: Municipalidad Metropolitana de Lima. 
cuando se derribaron las murallas y la ciudad se expandió hacia el Sur, con lo cual empezó la transición hacia la Lima moderna.

La segunda mitad del siglo XIX y la primera del XX son testigos del crecimiento urbano de la capital y de la intensificación de su depredación ambiental. El transporte a vapor de buques y trenes no solo permitió conectar el territorio nacional a velocidades nunca antes alcanzadas, sino que significó la depredación de las grandes áreas forestales remanentes, como las lomas de Villa María del Triunfo y Pachacamac, poseedoras de una abundante flora y fauna. Estas lomas alojaban bosques de árboles frutales y medicinales (como la lúcuma y el mito) que, en conjunción con la gran humedad invernal, contribuían a la formación de lagunas y riachuelos que desembocaban en el río Lurín, a través de las quebradas, y daban continuidad a su cauce durante todo el año (Chipana, 2013). Las lomas costeras fueron el blanco perfecto de los leñadores que abastecían las locomotoras de la ruta Lima-Lurín: fueron deforestadas por completo, y más tarde empresas cementeras exterminaron su biodiversidad en gigantescas áreas de terreno. Sin árboles ni otros elementos bióticos, los riachuelos y puquiales pronto acabaron por extinguirse. Curiosamente, la difusión del imaginario urbano de Lima como desierto se da recién hacia el siglo XX.

En cuanto al suelo de Lima, en la actualidad este es $19 \%$ arenal, $28 \%$ valle y $53 \%$ cordillera (roca). ¿Podríamos deducir, entonces, que Lima es más andina que costeña? Si consideramos al departamento de Lima en su conjunto, la proporción de cordillera aumenta.

¿Debemos, entonces, seguir entendiendo a Lima como su ambiente construido, o más bien deberíamos comenzar a incorporar la variable geográfica y territorial en la ecuación?

Es claro que, desde la visión de ciertas élites profesionales, Lima equivale a una porción de territorio construido y desertificado y, recientemente, también a la porción real de desierto: aquel $19 \%$ al norte de Ancón y al sur de Punta Hermosa. Ni qué decir de discursos aún más pintorescos, que buscan justificar una eterna clasificación de la realidad desde cerradas categorías occidentales y desde contextos urbanos únicamente. Los cerros y los remanentes de los valles, es decir, el ámbito rural de Lima, no terminan siendo nunca parte de la fórmula oficial, la cual curiosamente deja de lado a más de la mitad del espacio geográfico de la capital.

Aparentemente, la imponente presencia de aquellos colosos pétreos que marcan el inicio de la cordillera de los Andes hacia el este de la ciudad pasó desapercibida. La temporalidad, elemento olvidado al clasificar el espacio como desértico, le juega otra mala pasada al discurso de ciudad-desierto, al cubrir los cerros limeños de abundante vegetación y fauna cada año, durante la mitad del mismo. Ya lo notaba Charles Darwin en sus viajes por América del Sur, una mañana a mediados de julio, al inicio de la temporada de lomas costeras: "En las montañas cercanas a Lima (...) el suelo está cubierto de musgo y camas de preciosas flores amarillas Ilamadas Amancaes" (1860, p. 391).

Una suerte de simbiosis andino-costeña ya había sido destacada anteriormente por antropólogos y arqueólogos diversos para caracterizar el territorio de Lima:

A pesar de que las precipitaciones en forma de garúa son escasas en la costa central, esta es la zona más húmeda de la costa. Existen lomas estacionales, como las colinas de Ancón, CarabayIlo, Atocongo, Manchay, Asia, entre las más notables, hacia donde bajan los pastores de altura durante la estación seca de la sierra. Dentro de estas condiciones, las relaciones transversales entre la costa y la sierra central son igualmente significativas. (Matos Mendieta, 1980)

Costa y Sierra como parte de un mismo sistema territorial, como una unidad indivisible sin segregación aparente (Del Castillo, 2015). 


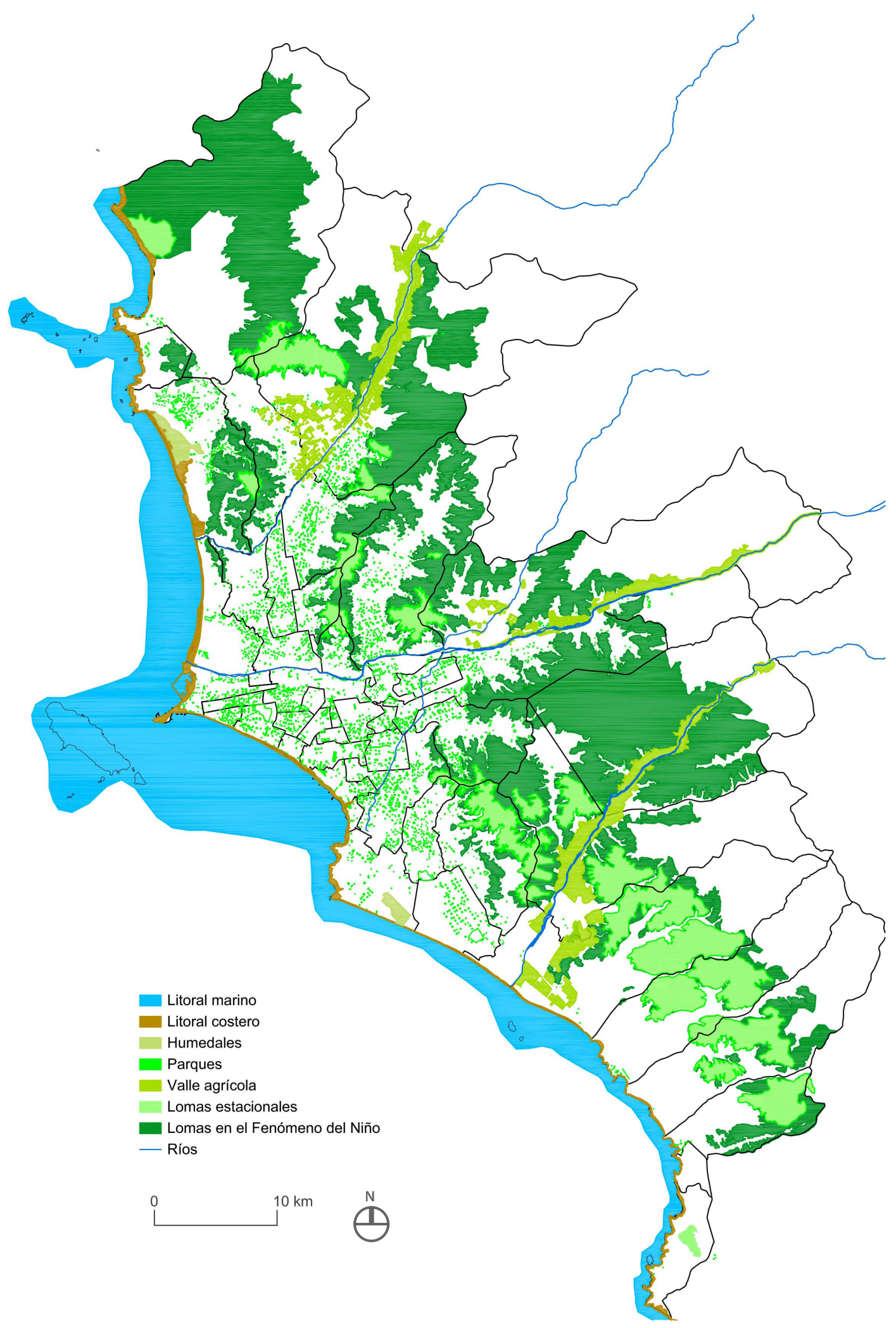

Figura 2. Lima y su Estructura Ecológica

Fuente. Elaboración propia a partir de Municipalidad Metropolitana de Lima (2014). Plan Metropolitano de Desarrollo Urbano Lima-Callao 2035. Lima, Perú: Municipalidad Metropolitana de Lima 


\section{Contexto del PLAM 2035}

Históricamente, el Plan Metropolitano de Desarrollo Urbano Lima-Callao 2035 es el cuarto plan que se ha propuesto para la ciudad de Lima, tras el Plan Piloto de Lima de 1949, el Plan de Desarrollo Metropolitano Lima-Callao Esquema Director 1967-1980 y el Plan de Desarrollo Metropolitano de Lima-Callao PLANMET 1990-2010.

Desde la publicación en 1992 del PLANMET, este sufrió una rápida desactualización, debido al momento de transición política y económica, el cambo de un modelo de intervención estatal a uno de Estado abiertamente neoliberal. Ya que la lógica de planeamiento tradicional de las propuestas específicas se apoyaba en la visión de un Estado gestor, el giro del sistema político hacia una economía de mercado y privatización de servicios públicos dejaba sin piso al aparato de gestión necesario para materializar el plan. Esta naturaleza dual del posicionamiento del plan también mermó de forma significativa sus posibilidades de llevarse a cabo, ante una coyuntura en la que dejar al mercado la solución de todos los problemas socioeconómicos y de infraestructura pública parecía ser la fórmula mágica del éxito.

Luego de casi 25 años después del inicio de este proceso desregulador en el Perú, al constatar que la planificación urbana ha sido reducida a la actualización de los usos de suelo que la dinámica frenética de la ciudad imponía a sus planificadores, asistimos a un escenario de nuevos retos. Entre ellos está el asegurar el abastecimiento de áreas de equipamiento adecuado para enfrentar la densificación que demanda la ciudad y una expansión autosostenible, y dejar atrás la extendida práctica del zoning monofuncional. Además, es crucial la incorporación de redes de ciclovías como infraestructura sostenible en la reforma del transporte y articular el cuantioso patrimonio histórico de la ciudad en una red espacial que permita su reconocimiento. También está el desafío de convertir el trinomio policentralidad-descentralización-integración regional en una alternativa realmente viable para Lima. Así mismo, hace falta la creación de nuevos espacios públicos que puedan satisfacer la creciente demanda de la población, no solo en zonas periféricas, donde más se necesitan, sino también en el área central metropolitana, lo cual implica una adecuada política de expropiaciones. Por último, es necesario incorporar una gestión de riesgos en la planificación de la ciudad, la experiencia participativa de la ciudadanía y una adecuada gestión medioambiental que permita la regeneración de los ecosistemas urbanos.

El PLAM2035 se enmarca, en el ámbito legal, dentro de los instrumentos técnicos para el ordenamiento territorial del país, que incluyen a su vez a los Planes Nacionales, Planes Sectoriales, Planes de Desarrollo Regional y Planes de Ordenamiento Territorial Regionales. Así mismo, se encuentra dentro de la estructura de planes urbanos especificados en el DS-004-2011 del Ministerio de Vivienda, mientras que a nivel municipal está dentro de la Ordenanza $\mathrm{N}^{\circ} 620$, que establece sus antecedentes y sus componentes. Este plan constituye una innovación en el campo de la planificación en el Perú, pues aborda por primera vez detalladamente temas como un diagnóstico medioambiental y la gestión de riesgos, además de incorporar una cartografía metropolitana actualizada del patrimonio edificado de Lima.

El documento contiene también un diagnóstico temático en el cual, además de elaborar un análisis actualizado de los aspectos social, medioambiental, económico y de movilidad, vivienda, equipamientos, espacios abiertos, servicios básicos, gestión de riesgos de desastres, gobernabilidad y patrimonio edificado, presenta una cartografía que traduce el análisis al nivel físico-territorial y pretende servir como base para un sistema de información de la ciudad. A nivel de propuesta, el documento organiza el territorio de la capital a partir de cuatro sistemas urbanísticos principales -Sistema de movilidad, Sistema de equipamientos, Sistema de espacios abiertos e infraestructura ecológica, y Sistema de servicios básicos- y dos redes urbanísticas funcionales -Red de centralidad, y Red de patrimonio y paisaje- (Municipalidad Metropolitana de Lima, 


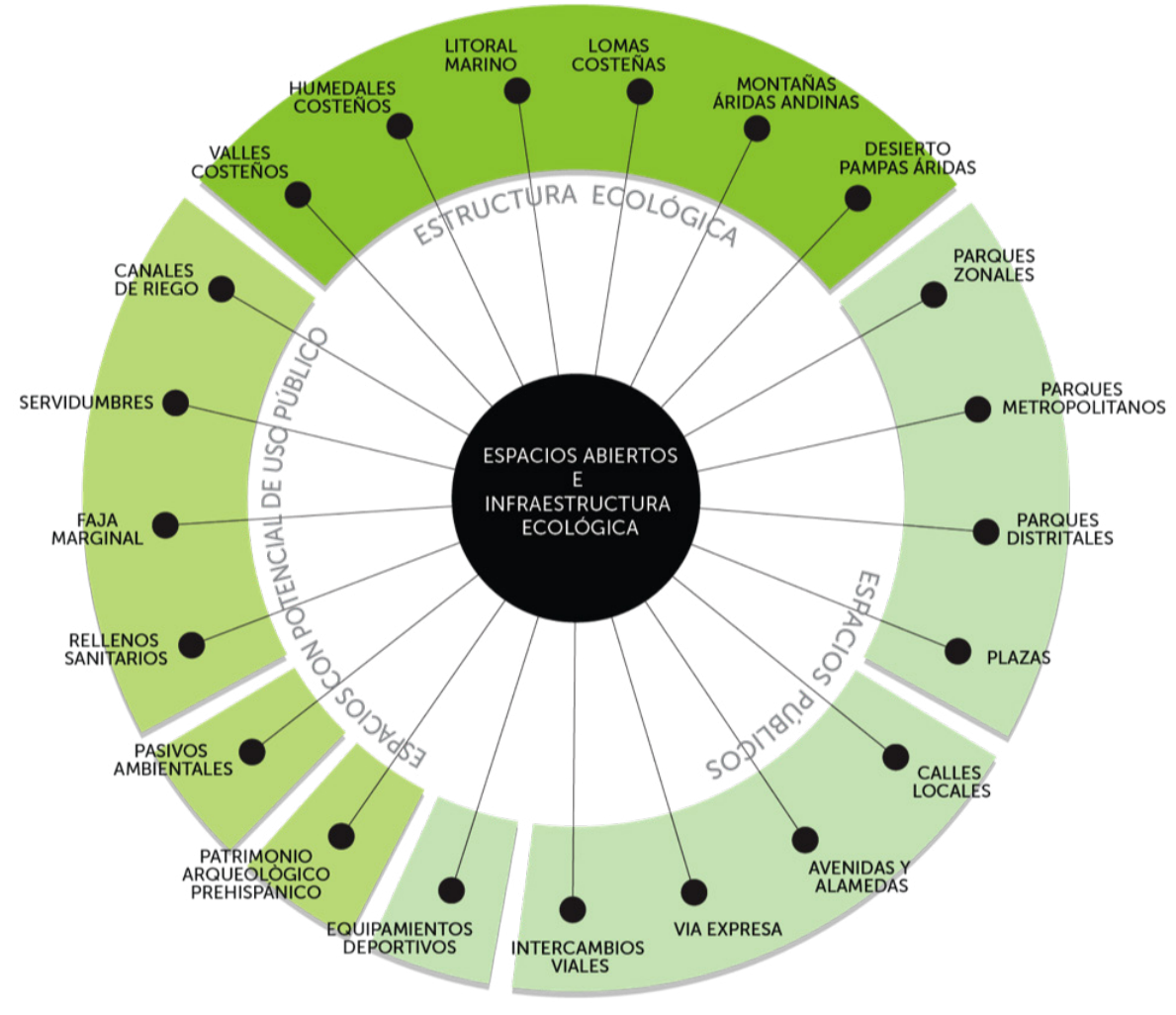

2014). Es justamente de la integración del Sistema de espacios abiertos e infraestructura ecológica (SEA+IE), y la Red de patrimonio y paisaje (RP+P) que surge la propuesta de vincular al patrimonio arqueológico y los espacios abiertos de tal forma que se promueva la protección del patrimonio, y a su vez se generen espacios públicos de calidad para el beneficio de la comunidad.

\section{Espacios abiertos. Una nueva visión}

El Sistema de espacios abiertos e infraestructura ecológica (EA e IE) tiene su origen en la importancia que otorga el PLAM2035 a los espacios públicos como generadores de ciudad. En planes previos, se había utilizado conceptos como área libre o área verde, los cuales se ven limitados en definición, ya que no garantizan el uso público ni reconocen las diferentes tipologías de espacios presentes en Lima Metropolitana.

La propuesta del sistema de EA e IE requirió de una reflexión acerca del concepto de espacio público aplicado a la ciudad de Lima. Actualmente, el Ministerio de Vivienda, por medio del DS-004-2011, solo considera a los espacios zonificados (Zonificación de Recreación Pública, ZRP) como espacios públicos, lo cual restringe el significado de lo público. Los espacios ZRP, por competencia legal se desarrollan solo en áreas urbanas o de expansión urbana, cumplen fines básicamente recreativos e incluyen una limitada cantidad de tipologías (plazas, parques, campos deportivos, juegos infantiles y similares): se deja de lado a espacios naturales en áreas no urbanizables o en áreas rurales, así como a espacios con potencial de uso público. Aunque socialmente los espacios públicos están entendidos como todos aquellos espacios de uso público, libres, irrestrictos, accesibles y flexibles sin limitaciones legales, de gestión o de propiedad del suelo, las definiciones reglamentarias, bajo la mirada de la zonificación, exponen una deficiencia legal que solo permite una comprensión sesgada de estos espacios en la ciudad.
Figura 3. Espacios abiertos e infraestructura ecológica. Tipos de espacio considerados

Fuente. Plan de Espacios Abiertos e Infraestructura Ecológica. Municipalidad Metropolitana de Lima y Servicio de Parques de Lima, 2014. p. 14 
A luz de una ciudad como Lima, que crece con lógicas individualistas y se desvincula cada vez más de sus espacios naturales, en el PLAM2035 se buscó utilizar un concepto amplio que dé importancia al valor de lo público, que reconozca la diversidad de espacios naturales en la ciudad y que permita integrar otros espacios que, aun sin estar incluidos en las definiciones legales, presenten potencial como espacios de integración ciudadana. El término 'espacios abiertos', en el PLAM2035, apuesta por integrar tres conceptos: espacios públicos, estructura ecológica y espacios con potencial de uso público. Además, considerando que el ser humano en el espacio público establece una relación tanto con otros seres humanos como con su entorno físico, se consideró también el término 'infraestructura ecológica' para referirse a los espacios abiertos que dan soporte ecológico a la ciudad, y permiten proteger y conservar ecosistemas, así como brindar servicios ambientales, sociales y económicos a la población.

A partir de esta nueva definición, el PLAM2035 elabora análisis cuantitativos y cualitativos de las tipologías comprendidas en estos tres grandes conceptos. Se mencionarán algunas conclusiones para poder entender las relaciones planteadas en dicho plan entre los espacios abiertos y el patrimonio arqueológico.

\section{Espacios Públicos}

Las tipologías consideradas en el PLAM2035 en cuanto a los espacios públicos en Lima incluyen las calles locales; las avenidas y alamedas; las vías expresas; los intercambios viales (óvalos, tréboles y puentes peatonales); las plazas; los parques distritales, metropolitanos y zonales; y los equipamientos deportivos abiertos con vocación de espacios público.

Un espacio importante que se integra al concepto de espacio público es la calle, cuyas cualidades como espacio de encuentro y socialización se busca resaltar. Es actualmente el espacio de mayor extensión (24 mil hectáreas) y el que más se identifica con la experiencia urbana cotidiana; sin embargo, se entiende hoy solo en función a sus características como espacio de tránsito (mayoritariamente vehicular) y es posible observar una gradual disminución de las dimensiones de las veredas (antes de entre 2 y 4 metros, y hoy de 1.20 o incluso 1 metro de ancho) y una consecuente incapacidad para desarrollar otros medios de movilidad alternativa.

En relación a las áreas verdes públicas en Lima, ha disminuido el índice de $\mathrm{m}^{2}$ por habitante (desde 1920, con $17.27 \mathrm{~m}^{2} /$ hab, hasta la actualidad, con $3.7 \mathrm{~m}^{2} /$ hab. en promedio), así como la presencia de parques de gran escala (hoy en día cerca de tres millones de habitantes no cuenta con un parque de escala zonal -más de 2 hectáreas- a una distancia de 2 mil metros de su vivienda) (Municipalidad Metropolitana de Lima, 2014). Los espacios de sociabilización y de contacto con la naturaleza brindan beneficios psicosociales y ambientales, y a su vez permiten encuentros ciudadanos, de interacciones tanto directas como indirectas, que facilitan el reconocimiento ciudadano y la pertenencia a la ciudad.

En cuanto a las plazas, en la ciudad su presencia se relaciona mayormente a losas-plazas en distritos emergentes ( $46 \%$ del total de plazas en la ciudad), canchitas de fulbito multifuncionales en las que se llevan a cabo desde manifestaciones religiosas o cívicas hasta campeonatos deportivos de barrio. Sin embargo, la cobertura de plazas a distancias cortas de las viviendas es todavía muy baja: en un radio de 300 metros, cerca de 6 millones y medio de habitantes no accede a una plaza en cercanía (Municipalidad Metropolitana de Lima, 2014).

\section{Estructura Ecológica}

La estructura ecológica de la metrópoli de Lima-Callao abarca 196 mil hectáreas, y comprende una diversidad de ecosistemas naturales y antrópicos que están en proceso de regresión y deterioro, tanto en extensión como en calidad. Le aporta a la ciu- 
dad servicios ambientales, sociales y culturales que por primera vez se integran a la planificación urbana. Esta se ha incluido en el PLAM2035 considerando dentro de sus tipologías a los valles costeños, los humedales, el litoral marino, las lomas costeñas, las montañas áridas, el desierto y las pampas áridas.

Los valles costeños, por ejemplo, abarcan en total 11 mil hectáreas, y representan el $27 \%$ de la cobertura vegetal de la ciudad; sin embargo, el crecimiento urbano amenaza con desaparecerlos. Por otro lado, las lomas, ecosistemas biomorfológicos secos (19 en 13 distritos) que se desarrollan en las zonas de neblinas invernales y aparecen todos los años entre julio y diciembre, abarcan 21 mil hectáreas y pueden llegar a cubrir, según las condiciones climáticas, hasta 69 mil hectáreas. Actualmente son ecosistemas frágiles cuyos límites y dimensiones han sido alterados por el proceso de expansión urbana, que ha generado un impacto negativo sobre la diversidad biológica y los restos arqueológicos presentes en ellas. Las lomas se encuentran en constante amenaza por la poca capacidad, demostrada hasta el momento, para relacionar los espacios naturales a los urbanos.

\section{Espacios con Potencial de Uso Público}

Las tipologías que el PLAM2035 considera dentro de esta clasificación son el patrimonio arqueológico prehispánico, los pasivos ambientales, los rellenos sanitarios, la faja marginal, los canales de riego y los espacios llamados servidumbres (espacios libres que acompañan a las infraestructuras de transporte, eléctricas, etc.). Para establecer la Red de patrimonio y paisaje en el PLAM2035, se ha contemplado el patrimonio arqueológico, el patrimonio histórico colonial y republicano, y los bienes contemporáneos con valor patrimonial. Por otro lado, para articular esta red con el Sistema de espacios abiertos e infraestructura ecológica, se ha considerado al patrimonio arqueológico.

Para comprender la categoría de patrimonio arqueológico prehispánico cabe mencionar que el patrimonio, según los investigadores Josep Ballart Hernández y Jordi Juan Treserras (Hayakawa, 2010), es aquello que proviene de los padres, bienes que hemos heredado y a su vez traspasamos en herencia. Su importancia está relacionada al recuerdo, la memoria y la identidad. Para dichos investigadores, el patrimonio establece una relación histórica ligada a actitudes creativas del pasado que nos conducen a la realidad actual; se pasa del monumento soporte de memoria al patrimonio soporte de identidad. Así, la relevancia del patrimonio arqueológico prehispánico tiene que ver, entre otras cosas, con el marco social, histórico y cultural de nuestras vidas en tiempo y espacio, en relación a todos los bienes edificados heredados antes de la llegada de los españoles al Perú (hasta el siglo XVI).

El patrimonio arqueológico prehispánico de Lima Metropolitana está conformado por 361 bienes que permiten conocer cómo se habitaba en el pasado el territorio sobre el cual se asienta nuestra ciudad. Estos bienes se dividen en categorías como conjuntos edificados, edilicia especial, fragmentos de construcciones, sitio natural y otros, y han sido agrupados en el PLAM2035 en tres tipos de conjuntos: cultural central (en áreas interdistritales de Lima Centro), cultural a repotenciar (en el área urbana del Callao, el Centro Histórico de Lima, y algunos sectores de Lima Norte y Sur) y corredor cultural (fuera del área central, cerca a los tres ríos que atraviesan la ciudad). Del total, 266 tienen definida su área a través de polígonos, mientras que el resto se encuentran mapeados solo como puntos en las bases de datos del Ministerio de Cultura y la Gerencia de Cultura de la Municipalidad de Lima (Municipalidad Metropolitana de Lima, 2014).

El patrimonio arqueológico en Lima está constantemente amenazado por la débil relación que los diferentes actores de la ciudad han mantenido con estos espacios a lo largo del tiempo. El crecimiento de la ciudad muestra una aparente imposibilidad por coexistir equilibradamente con sus espacios patrimoniales; se observa una predominancia del crecimiento y la expansión urbana que debilita sus conexiones 
urbanas y territoriales. Los espacios patrimoniales en Lima sufren de abandono, falta de interés, desconocimiento y despreocupación por parte de la sociedad, la inversión privada y el Estado.

Esto se evidencia, por un lado, en la falta de políticas públicas en relación a la protección, conservación y valoración del patrimonio arqueológico. Según la ley 28296 (Ley General del Patrimonio Cultural de la Nación), los sitios arqueológicos se delimitan de acuerdo a la extensión que técnicamente el Ministerio de Cultura considere adecuada para su protección. El área de protección es un área intangible, dentro de la cual se puede destinar espacio para uso público, tratamiento de paisaje, o acondicionamiento turístico o cultural. En la disposición complementaria final cuarta del nuevo Reglamento de Investigaciones Arqueológicas (DS 003-2014/MC) publicado en octubre de 2014, se indica que se reglamentarán los criterios técnicos para definir el marco circundante a los espacios patrimoniales; sin embargo, no se define con claridad qué implica esta definición. Por lo tanto, aunque exista la intención de generar definiciones de protección y se designen entidades responsables, legalmente el espacio de protección del patrimonio arqueológico prehispánico no existe. Si a esto se le adiciona el poco presupuesto que se asigna a la conservación y mantenimiento del patrimonio, resulta claro que la realidad de estos espacios es de desprotección y vulnerabilidad.

Por otro lado, al ser el suelo uno de los bienes más preciados en las ciudades y al no existir una política que garantice suelo público en Lima Metropolitana, los espacios patrimoniales prehispánicos en la ciudad son espacios apetecibles para invadir o urbanizar. Los proyectos de urbanización en Lima Metropolitana, promovidos por el sector privado y aprobados por el gobierno, así como las invasiones informales, han sido actores principales en el proceso de degradación del patrimonio. Esto se evidencia en procesos de urbanización ocurridos durante diferentes momentos de la historia de Lima: por ejemplo, el crecimiento de Miraflores y su impacto en la Huaca Pucllana, o la fragmentación de los espacios patrimoniales del Complejo Maranga en San Miguel. También las invasiones, con ocupaciones de borde o superpuestas al patrimonio arqueológico, denotan poco interés y/o desconocimiento de la población ante los espacios patrimoniales. Estos son vistos como montículos de tierra o como terrenos baldíos.

Esta situación genera preguntas en diferentes niveles: ¿Cómo comunicamos y comunicaremos en el futuro nuestra historia en la ciudad? Medidos por nuestros bajos niveles de responsabilidad y respeto patrimonial, ¿qué tipo de sociedad estamos formando?; ¿Cómo podría incrementarse nuestro nivel de conciencia frente al patrimonio arqueológico?; ¿qué tipo de ciudad sería Lima si, revirtiendo la tendencia actual de crecimiento en torno a centros comerciales, pensáramos en una ciudad cuyo desarrollo urbano se da contemplando a las huacas como generadoras de centralidad?; ¿qué lógicas de densidad (entendiendo que al densificar indefectiblemente se hace necesaria la creación de espacios abiertos públicos), podrían darse reconociendo las características del patrimonio de cada uno de los conjuntos culturales definidos por el PLAM2035?

Aunque las lógicas urbanas actuales denotan una apropiación negativa por parte de la población, también existen ejemplos de apropiación positiva que responden a la relación del patrimonio con algunas comunidades. Esto sucede, por ejemplo, cuando se generan vínculos culturales con el patrimonio, como puede ser la manifestación de tradiciones ancestrales (Inti Raymi) en la Huaca Fortaleza de Campoy en San Juan de Lurigancho, o cuando se vinculan actividades cotidianas a la preservación del patrimonio, como en la Huaca Pucllana. La identificación adecuada de actores a escala local y la correcta inserción del patrimonio arqueológico prehispánico a dinámicas contemporáneas de ciudad y territorio permitirán que los espacios patrimoniales y los espacios naturales estén integrados, y formen parte de nuestro sistema público, tanto físico como simbólico, brindando sentido y valor contemporáneo a estos espacios. 


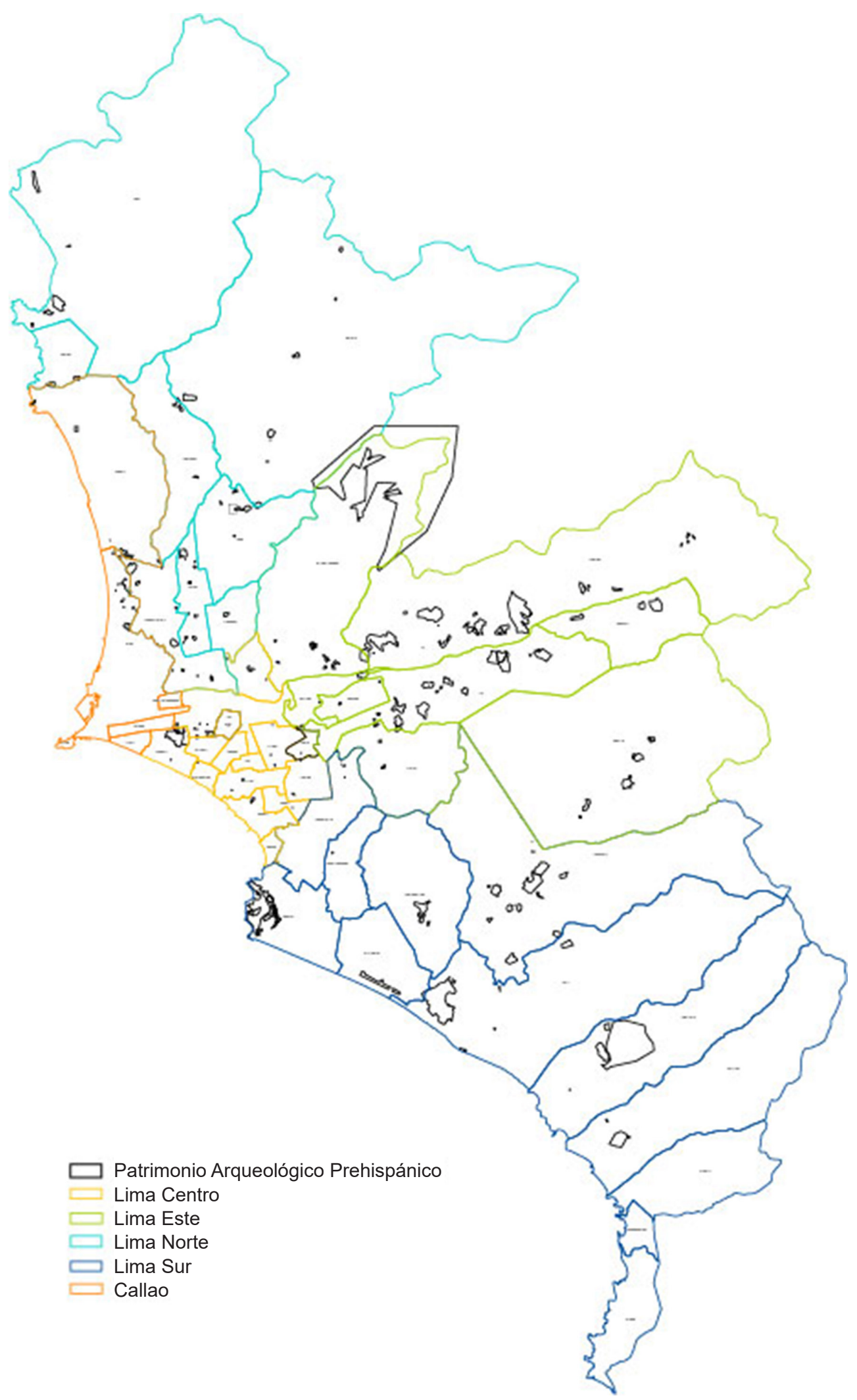

Figura 4. Patrimonio arqueológico prehispánico. Polígonos delimitados por el Ministerio de Cultura

Fuente. Información brindada al PLAM por el Ministerio de Cultura, 2014 


\section{Sistema de Espacios Abiertos e Infraestructura Ecológica}

En Lima Metropolitana, por muchos años los espacios públicos se han entendido de manera aislada, sin considerar las relaciones entre estos ni su rol en las dinámicas de la ciudad. En el PLAM2035 se define el Sistema de espacios abiertos e infraestructura ecológica como el conjunto de espacios al aire libre de uso público, y de propiedad y/o gestión pública o privada comprendidos por los espacios construidos, semiconstruidos o naturales ubicados en el área urbana, de expansión urbana, o en alguna de las unidades ambientales de la estructura ecológica de la ciudad de Lima. Los espacios abiertos públicos mejoran las condiciones de habitabilidad, dotando a la ciudad de espacios para el desarrollo humano y la construcción de ciudadanía, donde las regulaciones sociales se definen por los deberes y derechos ciudadanos en los que el bien común prevalece por sobre el bien individual. Son espacios que permiten actividades recreativas, de deporte, de esparcimiento, de comunicación, de movilidad e interacción social, que fomentan la realización de acciones cívicas y el ejercicio de la ciudadanía, entre otros. Este sistema está compuesto por seis subsistemas: calles, áreas verdes, áreas verdes complementarias, plazas, playas y red hidrológica.

De estos seis subsistemas, las calles y las áreas verdes (y sus tipologías, corredores verdes, parques culturales, etc.) se vinculan con el patrimonio arqueológico prehispánico a través de estrategias en Lima Metropolitana.

\section{Estrategias en el PLAM2035 para la Integración del Sistema de EA e IE, y el Patrimonio Arqueológico Prehispánico}

El patrimonio arqueológico prehispánico, en relación a la forma de crecimiento urbano de Lima, podría caracterizarse en dos grandes grupos: fragmentos de construcciones y conjuntos edificados. El patrimonio arqueológico que tuvo mayor relación con el crecimiento de la ciudad después de que se derrumbaran las murallas, y que fue absorbido por la ciudad en los distritos hoy son conocidos como céntricos o tradicionales, se encuentra hoy en día fragmentado, con muy poco o nulo espacio vacío de borde, como un espacio sitiado que ha tenido que pelear su lugar en la ciudad. En algunos distritos estos bienes patrimoniales han sido intervenidos para evitar su degradación y en algunos casos, como el de la Huaca Pucllana, Huallamarca o Huantinamarca, estos espacios han sido recuperados e integrados al paisaje urbano. Por otro lado, los espacios patrimoniales que quedaron ubicados en las afueras de la ciudad y que con el paso del tiempo se han integrado a los distritos de Lima Norte, Lima Este y Lima Sur se han mantenido más completos que los anteriores, sin perder su condición de grandes espacios arqueológicos. Esto se debe a que el proceso de consolidación en estas zonas, que empezó a manifestarse en la primera mitad del siglo XX, ha impactado menos en las dimensiones del patrimonio, lo cual ha permitido que tenga carácter de área patrimonial y no de fragmento. Sin embargo, debido a que no cuenta con políticas de protección, sufre de rápidos procesos de degradación.

Asumidas estas condiciones, el PLAM2035 plantea dos estrategias para integrar el patrimonio arqueológico a la ciudad. Por un lado, está la estrategia de asociatividad cultural que busca integrar a la dinámica urbana contemporánea el patrimonio arqueológico fragmentado en los distritos de Lima Central a través de la calle y los corredores verdes. Por otro lado, cuenta con la estrategia territorial cultural, realizable a través de los parques culturales. Esta última permite incorporar el concepto de paisaje cultural que la UNESCO define como el conjunto de obras que combinan el trabajo de la humanidad y la naturaleza.

\section{Estrategia de Asociatividad Cultural - Corredores Verdes}

Para que la estrategia de asociatividad cultural se lleve a cabo, se requiere la identificación e intervención de calles que vinculen espacios abiertos públicos con espacios 


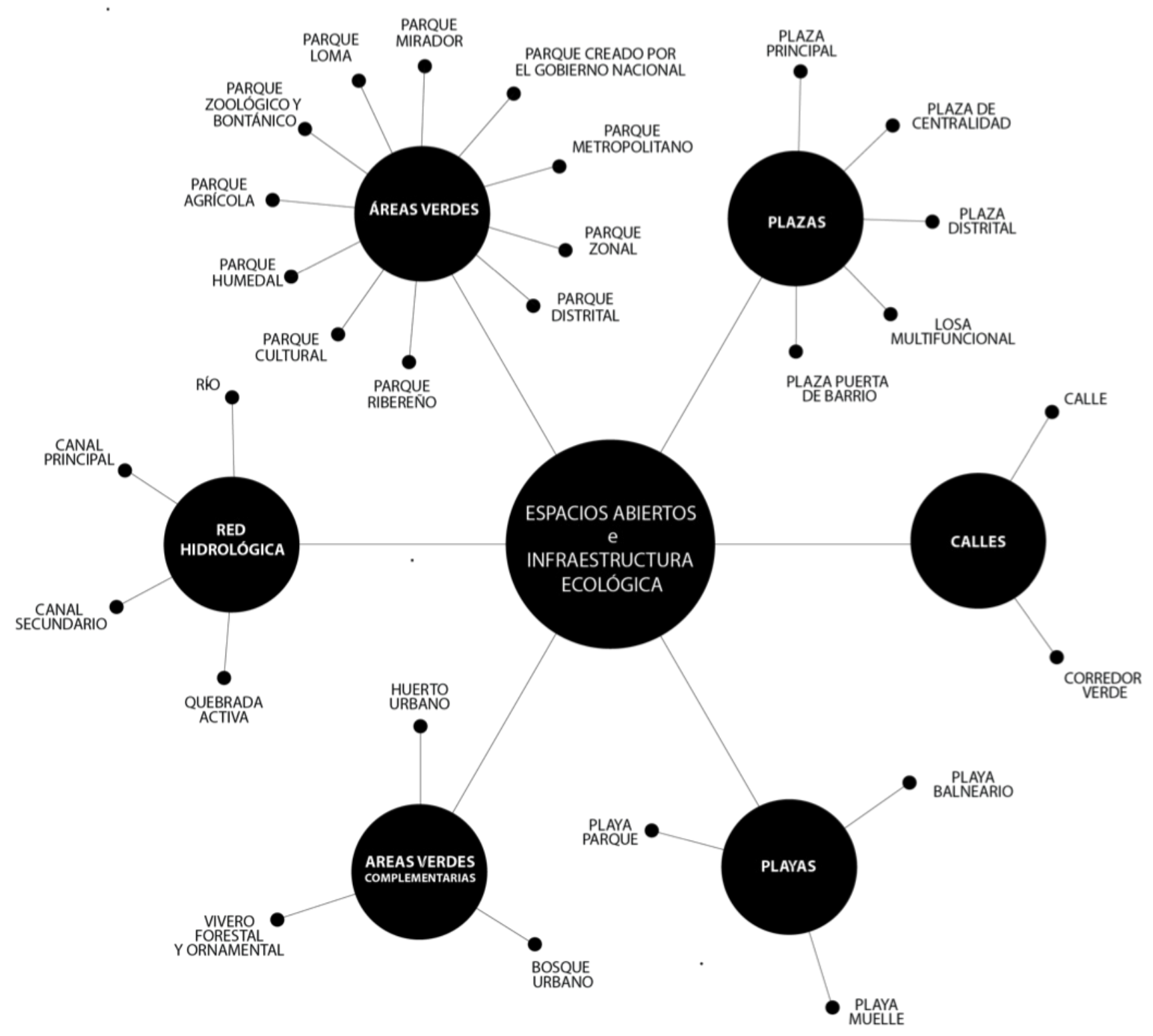

patrimoniales, caracterizados como fragmentos de construcción. Para lograrlo, se contempla la coordinación de tres sistemas (espacios abiertos e infraestructura ecológica, movilidad y equipamiento) con la Red de patrimonio y paisaje.

La primera acción para concretar esta coordinación es la identificación de las calles que permitirán el desarrollo del Sistema Integrado de Transporte Público, de tal manera que el espacio para los peatones y ciclistas aumente en la medida que disminuye el espacio para la circulación de vehículos privados. En segundo lugar, se contempla la mejora de la relación espacial entre las calles y espacios del tipo plazas-parques, de tal forma que desplazarse por la ciudad relacione de manera más eficiente a las tipologías de espacios públicos. Se considera también la identificación de calles próximas a los principales equipamientos en la ciudad, de manera que se mejore la experiencia urbana de acceso a los principales edificios públicos. Finalmente, una vez identificadas
Figura 5. Sistema de espacios abiertos e infraestructura ecológica

Fuente. Plan de Espacios abiertos e Infraestructura Ecológica. Municipalidad Metropolitana de Lima y Servicio de Parques de Lima, 2014. p. 178 
estas calles y sus espacios vinculantes, se busca su asociación física con el patrimonio arqueológico fragmentado, con el objetivo de generar una nueva red que permita articularlo, entendiendo que cada tipología de calle juega un rol en esta integración.

Las calles y avenidas de carácter metropolitano articularán el patrimonio a la estructura ecológica. Llamadas corredores verdes, constituirán redes que conectan y protegen recursos naturales y culturales, y cumplen con propósitos ecológicos, paisajistas, sociales, culturales, recreacionales, de movilidad y otros, compatibles con usos de suelo sustentable que permitan sostener una ciudad saludable a largo plazo (Plan EA e IE). Las calles locales con condiciones adecuadas, en cuanto espacios públicos, permitirán generar vínculos de escala barrial que integren el patrimonio a la vida cotidiana de los ciudadanos.

Los conjuntos culturales centrales y los conjuntos culturales a repotenciar se beneficiarán de estas articulaciones, gracias a la construcción de asociatividades a partir de las preexistencias. Algunas calles, vestigios de caminos prehispánicos y coloniales, podrían resignificarse e integrar la historia de la ciudad a lógicas urbanas actuales. Una nueva red de circuitos culturales podrá integrar las zonas patrimoniales a las dinámicas urbanas contemporáneas, tanto en áreas de regeneración urbana como en áreas culturales a reforzar.

\section{Estrategia Territorial Cultural - Parques Culturales}

Para que la estrategia territorial cultural se lleve a cabo se requiere la identificación de bienes patrimoniales en los corredores culturales (ligados a los tres valles de Lima), así como de ciertas condiciones urbanas que permitan el desarrollo de parques de gran escala.

Para lograrlo se identificó el patrimonio arqueológico en los tres corredores culturales (190 bienes) y se analizó aquellos que tienen polígonos definidos por el Ministerio de Cultura (60 bienes). Una vez identificados, se estudiaron los que cuentan con vacíos de borde considerable (1.5 hectáreas como mínimo), que permitan el desarrollo de grandes parques.

A esta identificación se aplicaron seis criterios de análisis en dos fases: los primeros cuatro estaban destinados a identificar los espacios factibles de intervención, y los últimos dos a establecer etapas de implementación. Los cuatro criterios iniciales están relacionados a la urgencia por conservar el patrimonio, a las posibilidades de uso público por su relación con áreas urbanas o centros poblados, la presencia de transporte público (actual y futuro) que garantice su accesibilidad y la cercanía a equipamiento metropolitano que permita que el uso del parque se realice de manera complementaria a otros espacios que brindan servicios en la ciudad. De esta forma se identificaron 22 parques culturales a implementar al 2035: diez en el corredor cultural del río Rímac, siete en el del río Lurín y cinco espacios patrimoniales fuera de los corredores culturales con espacios libres de 5 a 28 hectáreas que podrían destinarse a parques culturales.

Los dos últimos criterios de análisis, el déficit de espacios verdes públicos y plazas, y la densidad poblacional, dieron como resultado el planteamiento de la implementación de parques culturales en dos etapas. La primera, de corto y mediano plazo, aportaría 174 hectáreas de áreas verdes públicas a la ciudad, distribuidas en 11 parques culturales. La segunda etapa, de largo plazo, implicaría la implementación de 11 parques culturales adicionales, que sumarían 56 hectáreas más.

Los parques culturales son espacios abiertos públicos de escala metropolitana que buscan proteger el patrimonio arqueológico, desarrollar la vida pública, integrar el patrimonio a la estructura ecológica, disminuir el déficit de área verde por habitante, y brindar alternativas que permitan el encuentro de lo natural y lo urbano. Dentro del PLAM2035, surgen como una alternativa que busca la recuperación de la herencia cultural no solo a través de su restauración, y de evidenciar su valor y dinamizar la gestión del patrimonio, sino de su uso sostenible en espacios públicos con condiciones adecuadas de accesibilidad y seguridad, que promuevan la inclusión de diversos grupos sociales (Municipalidad Metropolitana de Lima, 2014). 


\section{Conclusión}

Es importante ser conscientes de que tanto los espacios abiertos como el patrimonio histórico de Lima se encuentran actualmente amenazados por la urbanización informal, y la falta de planificación y vivienda para los sectores populares, las cuales se traducen en invasiones promovidas por mafias de terrenos, proyectos inmobiliarios masivos, actividades extractivas y acumulación de basurales, entre otras actividades que vienen destruyendo los espacios de nuestra estructura ecológica y patrimonial ante el abandono del Estado respecto de su rol de ente regulador del crecimiento urbano.

Por otro lado, si bien es cierto que tanto la naturaleza urbana como las huacas se están convirtiendo poco a poco en escenarios para el activismo ecológico, indígena, deportivo e histórico (ciclistas, caminantes, escolares, peruanistas, etc.) de parte de la ciudadanía, que encuentra en ellos espacios de reencuentro con su identidad ancestral, su protección, regeneración y puesta en valor no podrá controlar y revertir los efectos de la urbanización insostenible que actualmente tiene lugar sin el apoyo de políticas públicas fuertes, que empoderen a estos grupos y promuevan sus actividades de forma masiva. Es muy importante tomar como referencia los numerosos estudios que vinculan el aumento de biodiversidad con la salud pública urbana, y generar nuevas investigaciones para sustentar la importancia de la conservación y regeneración de la estructura ecológica de la ciudad, y del patrimonio andino como huella ancestral de integración con la naturaleza y el manejo sostenible del territorio.

Los corredores verdes son fundamentales para esta tarea, pues cumplen el rol de unir la red patrimonial a la estructura ecológica y establecen, a su vez, corredores ecológicos para el libre tránsito de los flujos de biodiversidad. Si bien es cierto que el patrimonio arqueológico no ha tenido una participación muy activa en las lógicas de crecimiento urbano, los espacios patrimoniales necesitan formar parte de las dinámicas urbanas contemporáneas de tal forma que su presencia en la ciudad no solo se asocie a visitas turísticas esporádicas, sino que puedan ser reconocidos como espacios de oportunidad y desarrollo. Si se diseñaran modelos de negocio en torno al patrimonio, los cuales busquen integrar las estrategias de parques culturales y de corredores verdes a actividades económicas, y a nuevas formas de habitar la ciudad, se atesoraría la presencia del patrimonio como historia tangible integrada a la vida diaria, como espacio abierto donde se garantiza la vida pública y como espacio de oportunidad económica. Es necesario generar actividades productivas que consideren a los actores locales, a partir de asociar, por ejemplo, la agricultura de los valles de la ciudad a servicios campestres o gastronómicos con espacios abiertos a borde de río y en contacto con las áreas naturales.

Además, la integración de espacios patrimoniales en zonas centrales de la ciudad, con espacios de investigación, vivienda, y equipamiento educativo y cultural permitirín generar un nuevo tipo de apropiación cultural. La inversión privada, actuando en sociedad con el sector público, podría tomar parte en la conservación del patrimonio, de tal forma que habitar cerca de una huaca sea sinónimo de oportunidad, protección y desarrollo. Finalmente, el patrimonio arqueológico prehispánico debe poder integrarse al rediseño de nuestro modelo de ciudad, de tal manera que el desarrollo urbano asegure la coexistencia entre las dinámicas actuales y las pasadas, y las potencie en el futuro.

\section{Referencias}

Alberti, M., Marzluff, J., Shulenberger, E., Bradley, G., Ryan, C., \& Zumbrunnen, C. (2003). Integrating humans into ecology: Opportunities and challenges for studying urban ecosystems. BioScience 53 (12), 1169-1179.

Benítez, G., Pérez-Vásquez, A., Nava-Tablada, M., Equihua, M., \& Álvarez-Palacios, J. (2012). Urban expansion and the environmental effects of informal settlements on the outskirts of Xalapa city, Veracruz, Mexico. Environment and Urbanization, 24(1), 149-166. 
Buck, D. (6 de 12 de 2012). Stepping in the right direction: Giving Mother Earth rights. Recuperado el 27 de 12 de 2014, de Pachamama Alliance: http://www.pachamama.org/blog/stepping-in-the-right-direction-giving-mother-earth-rights

Buenaño, J. C. (2000). Historia y evolución de la ordenación forestal: Una aproximación. Investigaciones Sociales, IV (5), 181-186.

Chipana, J. (2013). Agricultura \& minería en una zona ecológica de Lima: Las Lomas de Atocongo (1912-1942). Lima, Perú: Instituto Ichsma.

Cieza de León, P. (1550). La crónica del Perú. Madrid, España: Historia 16.

Cook, N. D. (1981). Demographic collapse: Indian Peru, 1520-1620. New York: Cambridge University Press.

Darwin, C. (2014). A naturalist's voyage round the world: The voyage of the Beagle. New York: Skyhorse Publishing.

Del Castillo, J. M. (2015). Lima biotopo: Ecosistemas de montaña, patrimonio arqueológico indígena y activismo en los intersticios urbanos de la megalópolis andina. VII Seminario Internacional de Investigación en Urbanismo (p. 99). Montevideo, Uruguay: Universidad de la República de Uruguay-Universidad Politénica de Barcelona.

Elmqvist, T. (2013). Urbanization, biodiversity and ecosystem services: Challenges and opportunities. New York: Springer Open.

Harvey, D. (2000). Spaces of hope. Edinburgh, Scotland: Edinburgh University Press.

Hayakawa, J. (2010). Gestión del patrimonio cultural y centros históricos latinoamericanos: Tendiendo puentes entre el patrimonio y la ciudad. Lima, Perú: ANR.

Hayakawa, J. (2012). Restauro UNI: Breve antología de textos de textos de restauración del patrimonio monumental edificado. Lima, Perú: Universidad Nacional de Ingeniería.

Jorgensen, A., \& Tylecote, M. (2007). Ambivalent landscapes-wilderness in the urban interstices. Landscape Research, 32(4), 443-462.

Kaulicke, P. (2008). La economía en el período formativo. En W. Espinoza, L. G. Lumbreras, P. Kaulicke, \& J. I. Santillana, Economía Prehispánica, Tomo I (pp. 208-212). Lima, Perú: Banco Central de Reserva del Perú.

Lumbreras, L. G. (2008). Los orígenes de la sociedad andina. En W. Espinoza, L. G. Lumbreras, P. Kaulicke, \& J. I. Santillana, Economía Prehispánica, Tomo I (pp. 102-110). Lima, Perú: Banco Central de Reserva del Perú.

Matos Mendieta, R. (1980). Las culturas regionales tempranas. En Historia del Perú, Perú Antiguo, Tomo I (págs. 351-513). Barcelona, España: Juan Mejía Baca.

Municipalidad Metropolitana de Lima. (1992). Plan de Desarrollo Metropolitano de Lima-Callao 1990-2010. Lima, Perú: Municipalidad Metropolitana de Lima.

Municipalidad Metropolitana de Lima. (2014). Plan Metropolitando de Desarrollo Urbano Lima y Callao 2035. Lima, Perú: Municipalidad Metropolitana de Lima.

Pickett, S., Burch Jr., W., Dalton, S., Foresman, T., Grove, J., \& Rowntree, R. (1997). A conceptual framework for the study of human ecosystems in urban areas. Urban Ecosystems, 1(4), 185-199.

Riofrío, G., \& Cabrera, T. (2010). Expansión urbana y comunidades campesinas: Una entrevista a Erick Romero Mallqui. Quehacer, 179, 58-65.

Sagarin, R., \& Pauchard, A. (2012). Observation and Ecology: Broadening the scope of science to understand a complex world. Washington DC: Island Press.

Sandifer, P., Sutton-Grier, A., \& Ward, B. (2014). Exploring connections among nature, biodiversity, ecosystem services, and human health and well-being: Opportunities to enhance health and biodiversity conservation. Ecosystem Services, 12, 1-15.

Swyngedouw, E. (2010). Place, nature and the question of scale: Interrogating the production of nature. Berlín, Alemania: Berlin-Brandenburgische Akademie der Wissenschaften.

Ward Thompson, C. (2002). Urban open space in the 21st century. Landscape and Urban Planning, 60(2), 59-72. 